\title{
Image Processing and Imaging Technology in Cardiac Medical Surgery
}

\author{
Li Han $\mathbb{D}^{1}$ and Yun Yang $\mathbb{D}^{2}$ \\ ${ }^{1}$ Zhengzhou University of Light Industry, College of Computer and Communication Engineering, Zhengzhou 450001, \\ Henan, China \\ ${ }^{2}$ The Second Affiliated Hospital of Zhengzhou University, Zhengzhou 450000, Henan, China \\ Correspondence should be addressed to Li Han; hanli@zzuli.edu.cn
}

Received 22 July 2021; Revised 1 September 2021; Accepted 8 September 2021; Published 29 January 2022

Academic Editor: Gustavo Ramirez

Copyright (c) $2022 \mathrm{Li}$ Han and Yun Yang. This is an open access article distributed under the Creative Commons Attribution License, which permits unrestricted use, distribution, and reproduction in any medium, provided the original work is properly cited.

\begin{abstract}
In this thesis, transesophageal multiplanar ultrasound probes are used to detect epicardial echocardiography during cardiac valve replacement surgery. We observed the heart structure and valve function, left ventricular systolic function, residual shunt, and other information. There were three patients in the ultrasound examination before cardiopulmonary bypass. This has new findings and supplements for preoperative diagnosis. So, we changed the surgical approach and surgical plan and increased the surgical content. Epicardial echocardiography is easy to operate and has a wide range of indications. It has particular clinical application value in cardiac surgery.
\end{abstract}

\section{Introduction}

Clinical applications have recognized the value of echocardiography (EE) in cardiovascular surgery. There have been many studies on the role of transesophageal echocardiography (TEE) in intraoperative ultrasound diagnosis and efficacy judgment. Traditional epicardial ultrasound generally uses a sterile sheath-wrapped phased array transthoracic probe for inspection. In this study, a multiplane transesophageal ultrasound probe was used to perform epicardial ultrasonography during cardiac surgery [1]. We study the operating specifications and technical guidelines for intraoperative monitoring. Explore its specific application value and operation methods in a variety of cardiac surgery operations.

In recent years, the application of high-frequency ultrasound to examine the apex of the heart and the pericardium of the anterior wall of the right ventricle can accurately measure the thickness of the apex and the pericardium of the anterior wall of the right ventricle and can accurately identify the outer layer of the pericardium and a small amount of fluid in the pericardial cavity. Diagnosis has an important reference value.

\section{Materials and Methods}

2.1. Object. From March 2019 to November 2020, 53 patients underwent open-heart surgery in the cardiovascular department. Among them, the number of males is 33 and the number of females is 20 . The age ranges from 1 month to 70 years, and the median age is 47 years. All patients were routinely examined by conventional thoracic echocardiography before operation. The distribution of disease types and surgical methods are shown in Table 1. This article refers to the US GE-vivid7pvo color Doppler ultrasound system.

2.2. Apparatus and Methods. For intraoperative examination, we used the vivid color Doppler ultrasound imaging system from GE. During surgery, we use GE6T (adults) and 9T (children) transesophageal multiplanar ultrasound probes with a frequency of $3 \sim 8 \mathrm{MHz}(6 \mathrm{~T}) / 3 \sim 10 \mathrm{MHz}$ (9T). The intraoperative examination was performed after the cardiopulmonary bypass was opened and rebounded. Inspection methods include two-dimensional, spectral, and color Doppler imaging. Ultrasound operators have rich 
TABLE 1: The distribution of disease types and surgical methods in 53 patients with intraoperative transesophageal epicardial ultrasound (cases).

\begin{tabular}{|c|c|c|c|}
\hline Types of diseases and surgical procedures & $N$ & Types of diseases and surgical procedures & $N$ \\
\hline Mitral valvuloplasty & 7 & Balloon dilation of pulmonary valve stenosis & 2 \\
\hline Ventricular septal defect repair & 7 & Coronary artery right ventricular fistula repair & 1 \\
\hline Hypertrophic cardiomyopathy outflow tract dredging & 5 & Mechanical tricuspid valve replacement & 1 \\
\hline Mechanical aortic valve replacement & 5 & Correction of complete endocardial cushion defect & 1 \\
\hline Mechanical mitral valve replacement & 4 & Complete transposition of the great artery switch surgery & 1 \\
\hline Mitral valve bioprosthetic & 3 & $\begin{array}{l}\text { Pulmonary circumcision with complete transposition of the great } \\
\text { arteries }\end{array}$ & 1 \\
\hline Mitral valve + aortic valve mechanical replacement & 3 & Correction of tricuspid valve depression & 1 \\
\hline Tricuspid valvuloplasty & 2 & Aortic valve bioprosthetic & 1 \\
\hline Ventricular septal defect repair + patent arterial ligation & 2 & Ventricular septal defect repair + aortic arch angioplasty & 1 \\
\hline $\begin{array}{l}\text { Mechanical mitral valve replacement }+ \text { coronary artery bypass } \\
\text { grafting }\end{array}$ & 2 & Atrial septal defect repair & 1 \\
\hline Correction of subaortic stenosis & 2 & & \\
\hline
\end{tabular}

experience in transthoracic and transesophageal echocardiography. All patients underwent routine transthoracic ultrasound review 5-10 days after the operation [2].

2.2.1. Operation Method. We use a disposable sterile medical protective cover, and the protective cover and the probe are coupled with a coupling agent. The surgeon puts the transesophageal probe chip side protected by a sterile sleeve on the surface of the heart, and the surgeon completes the change of the attachment position. The sonographer holds the transesophageal probe and manipulates the instrument to obtain the best observation section by adjusting the scanning angle [3].

2.2.2. Commonly Used Cuts. There are different cutting methods in different operations. See Table 2, for standard cuts.

2.3. The Basic Model of Image Processing. Define the set $\Gamma=$ $\{1,2, \ldots, n\}$ of natural numbers. Suppose there is a set $R=$ $\left\{r_{1}, r_{2}, \ldots, r_{n}\right\}$ composed of $n$ rectangular elements with original dimensions. The size matrix of each rectangular piece $r_{i}$ is $r_{i}=\left[w_{i} h_{i}\right] . w_{i}, h_{i}$, respectively, represents the width and height of the $i$ rectangular piece. Assume that all dimensions are integers. The size, width, and height of the actual rectangular piece layout are $w_{r i}, h_{r i}(i \in \Gamma)$, respectively. It introduces the $90^{\circ}$ rotation variable $p_{i} \in\{0,1\}(i \in \Gamma)$, which satisfies the following relationship [4]: a collection of rectangular elements of the original size. Here, the random variable in the collection of elements is expressed:

$$
\left[w_{i} h_{i}\right]=\left[w_{i} h_{i}\right]\left[\begin{array}{cc}
1-p_{i} & p_{i} \\
p_{i} & 1-p_{i}
\end{array}\right]
$$

Assume that the lower-left corner of an unlimited rectangular container with width $W$ and height $H$ is the origin of the universal two-dimensional rectangular coordinate system. The horizontal and vertical directions of the container are, respectively, the $x$ - and $y$-axis. Let coordinate $\left(x_{i}, y_{i}\right)$ be the layout point of the $i$ rectangle.

The coordinate area established by the rectangular container is bounded by the side length of the $i$ rectangle. It is divided into four restricted areas $I, I I, I I I, I V$. Suppose that, in addition to any rectangular piece, $r_{i}=\left[w_{i} h_{i}\right](i \in \Gamma)$, there is also any rectangular piece $r_{j}=\left[\begin{array}{ll}w_{j} & h_{j}\end{array}\right](i \neq j, j \in \Gamma)$. Its layout point is $\left(x_{j}, y_{j}\right)$. To meet the restriction condition $b$, each area needs to meet:

$$
\left\{\left\{\begin{array} { l } 
{ x _ { j } + w _ { r j } \leq x _ { i } } \\
{ y _ { j } \geq 0 }
\end{array} I I \left\{\begin{array} { l } 
{ x _ { j } \leq x _ { i } \leq w _ { r i } } \\
{ y _ { j } + h _ { r j } \leq y _ { i } }
\end{array} I I I \left\{\begin{array} { l } 
{ x _ { i } + w _ { r i } \leq x _ { j } } \\
{ y _ { i } \geq 0 }
\end{array} I V \left\{\begin{array}{l}
x_{j} \leq x_{i} \leq w_{r i} \\
y_{i}+h_{r j} \leq y_{j}
\end{array}, \quad i, j \in \Gamma, i \neq j .\right.\right.\right.\right.\right.
$$


To satisfy the constraint $c$, any $r_{i}=\left[w_{i} h_{i}\right](\forall i \in \Gamma)$ only needs to satisfy

$$
x_{i}+w_{r i} \leq W,\left(x_{i}, y_{i}\right) \geq 0 .
$$

$$
\left\{\min H=\max \bigcup_{i=1}^{n}\left(y_{i}+h_{r i}\right)\right. \text { s.t. }
$$

$\left\{\begin{array}{l}\forall i \in \Gamma: \\ {\left[w_{r i} h_{r i}\right]\left[w_{i} h_{i}\right]\left[\begin{array}{cc}1-p_{i} & p_{i} \\ p_{i} & 1-p_{i}\end{array}\right](a)} \\ x_{i}+w_{r i} \leq W,\left(x_{i}, y_{i}\right) \geq 0(b) \\ \forall i, j \in \Gamma, i \neq j:(c) \\ x_{j}+w_{r i} \leq x_{i} \cup y_{j}+h_{r j} \leq y_{i} \cup \\ x_{i}+w_{r i} \leq x_{j} \cup x_{j} \leq x_{i}+w_{r i} .\end{array}\right.$

The layout points of all rectangular pieces in model (4) and the sum of their size. The maximum value of the union element is the final optimized layout height. s.t. (a) satisfies the restriction conditions in the problem description (a) and ensures that the rectangular part rotates freely at $90^{\circ}$. s.t. (b) satisfies the constraint (c) in the problem description and ensures that the layout of all rectangular pieces is in the rectangular container [5]. s.t. (c) satisfies the restriction condition (b) in the problem description and ensures that all rectangular pieces do not overlap each other.

\section{Results}

All 53 patients underwent transesophageal and epicardial ultrasonography after cardiopulmonary bypass, and all obtained satisfactory images. Six patients were found to have accumulated gas in the cardiac cavity after a standard exhaust after the open cardiopulmonary bypass. The gas was exhausted again until the gas disappeared. Four patients were found to have microbubble pneumatosis during the operation. The time of transesophageal epicardial ultrasonography was 3-7 min/time, with an average of $(4.2 \pm 1.4) \mathrm{min} /$ time. None of the 53 patients had complications such as infection, bleeding, or severe arrhythmia.

\subsection{Assessment of Segmental Wall Motion and Ventricular} Function. The ventricular wall motion and cardiac function of 53 patients were evaluated immediately after cardiopulmonary bypass. In 5 cases, it was found that the left ventricular wall movement was not coordinated, and the heart function was reduced. One patient with mechanical mitral valve replacement at the same time underwent coronary artery bypass grafting showed that the motion of the apex of the ventricular wall was reduced. This suggests that the blood flow of the anterior descending artery bypass graft is unsatisfactory. After re-bypass [6], the left ventricular wall motion recovered somewhat, and the left ventricular contraction motion was normal.
The ultimate goal of the two-dimensional orthogonal rectangular layout is to minimize the layout height $H$. Therefore, the following mathematical model is established:
3.2. Evaluation of Valve Surgery. One patient with mechanical mitral valve replacement showed residual paravalvular shunt immediately after the transesophageal epicardial ultrasound, and the residual shunt disappeared after another turnaround. The postoperative examination of the remaining 18 valve replacement patients showed that the mechanical or bioprosthetic valve position was expected. The valve opening and closing were standard, and there was no central and paravalvular regurgitation. This is consistent with the results of postoperative transthoracic ultrasonography (Figure 1) [7]. One case of tricuspid valve deformity showed moderate valve regurgitation after tricuspid valvuloplasty (Figure 2). The effect of re-transplantation was satisfactory. In 9 patients with valvuloplasty, the valve regurgitation was minimal. The valve orifice blood flow velocity was normal. Postoperative transthoracic ultrasonography was confirmed again. In 2 patients with pulmonary valve stenosis balloon dilatation, the balloon catheter was placed during the operation, and the dilatation effect was evaluated many times. This showed that the transvalvular pressure difference of the pulmonary valve was significantly reduced from the preoperative $78 \mathrm{mmHg}$ $(1 \mathrm{mmHg}=0.133 \mathrm{KPa})$ to $25 \mathrm{mmHg}$. And, the pulmonary valve regurgitation was small [8].

\subsection{Evaluation of the Effect of Congenital Heart Disease} Surgery. One patient with a ventricular septal defect repaired after an epicardial ultrasound showed a small amount of residual shunt (Figure 3). One case found suspicious ventricular horizontal residual shunt, and continuous Doppler observation of the spectrum was finally diagnosed as the residual shunt. All were successfully transferred again [9].

\subsection{Assess the Improvement of Left Ventricular Outflow Tract} Obstruction. Five patients with hypertrophic cardiomyopathy were measured immediately after the operation. In one case, it was found that the outflow tract blood flow rate was too fast. The flow rate was $3.2 \mathrm{~m} / \mathrm{s}$, which exceeded the normal range. After another transfer operation, the flow rate 
TABLE 2: Common views of transesophageal epicardial ultrasound.

\begin{tabular}{|c|c|c|}
\hline Section name & Main clinical applications & Probe position \\
\hline $\begin{array}{l}\text { Left long } \\
\text { ventricular axis }\end{array}$ & $\begin{array}{c}\text { Assessment of cardiac function, valve surgery to observe the } \\
\text { opening and closing of the aortic valve and mitral valve, and } \\
\text { hypertrophic cardiomyopathy surgery to observe the } \\
\text { thickness of the ventricular septum and left ventricular } \\
\text { outflow tract stenosis }\end{array}$ & $\begin{array}{l}\text { Right ventricular outflow tract near the front of the } \\
\text { heart and the angle is parallel to the long axis of the left } \\
\text { ventricle }\end{array}$ \\
\hline $\begin{array}{l}\text { The short axis of } \\
\text { the aorta }\end{array}$ & $\begin{array}{l}\text { Ventricular septal defect repair surgery to observe the } \\
\text { presence or absence of residual shunt and the location of the } \\
\text { residual shunt; aortic valve surgery to observe the condition of } \\
\text { the aortic valve }\end{array}$ & $\begin{array}{l}\text { Right ventricular outflow tract near the front of the } \\
\text { heart; the angle is perpendicular to the long axis of the } \\
\text { left ventricle }\end{array}$ \\
\hline $\begin{array}{l}\text { Left short } \\
\text { ventricular axis }\end{array}$ & Wall motion & $\begin{array}{l}\text { Right ventricular outflow tract near the front of the } \\
\text { apex; the angle is perpendicular to the long axis of the } \\
\text { left ventricle }\end{array}$ \\
\hline $\begin{array}{l}\text { Apical four- } \\
\text { chamber heart }\end{array}$ & $\begin{array}{l}\text { Ventricular wall motion, the opening, and closing of the } \\
\text { second and tricuspid valves }\end{array}$ & $\begin{array}{l}\text { The diaphragm surface at the apex of the heart, showing } \\
\text { the left and right ventricles and the two and tricuspid } \\
\text { valves }\end{array}$ \\
\hline
\end{tabular}

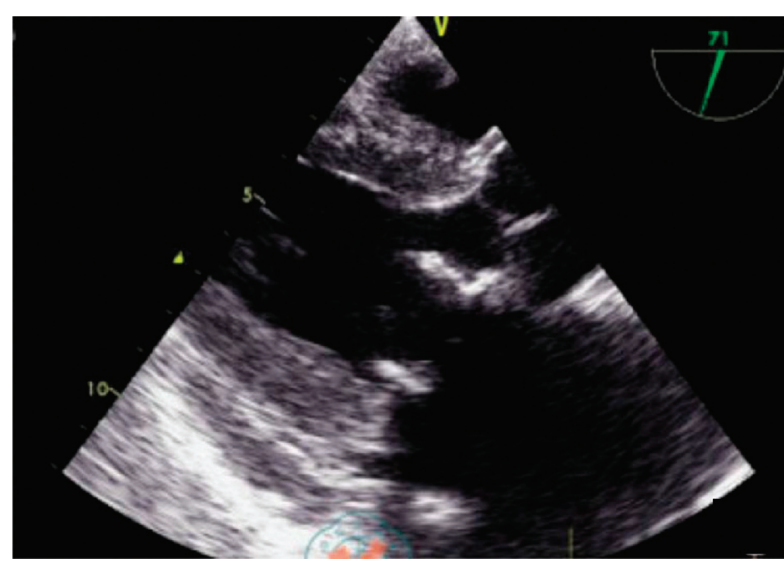

FIgURE 1: Two-dimensional ultrasound left ventricular long-axis view shows that the bioprosthesis is well opened and closed after the mitral valve bioprosthesis, and the left ventricular function is normal.

dropped to $1.4 \mathrm{~m} / \mathrm{s}$, and the pressure difference was $8 \mathrm{mmHg}$, and the effect was satisfactory. The pressure difference is less than $16 \mathrm{mmHg}$ (Figure 4).

\section{Discussion}

In recent years, the advancement of cardiac surgery diagnosis and surgical technology has put forward more and higher requirements for intraoperative ultrasound monitoring. According to the needs of surgery, transesophageal echocardiography and epicardial echocardiography are used for intraoperative monitoring. Transesophageal echocardiography has been widely used for intraoperative monitoring, guidance, and evaluation of cardiac surgery. Large-scale studies have confirmed that the application of epicardial echocardiography to monitor cardiac function. And, surgical results under thoracotomy conditions are a noninvasive and effective method. The probe is directly placed on the surface of the heart to avoid various interferences in the path of the sound beam. At the same time, due to the use of relatively high-frequency probes, the image is more transparent, and it can select multiple slices individually. An essential is that epicardial ultrasound has unique application value in the following situations. (1) Emergency abnormalities during cardiac surgery, such as weak contraction, reduced cardiac output, and severe recurring arrhythmia when the heart re-beats: in this study, an epicardial ultrasound in a patient with mitral valve mechanical valve replacement and coronary artery bypass grafting simultaneously detected abnormal left ventricular wall motion and decreased cardiac function. This suggests that the blood flow of the coronary artery bypass is not satisfactory, and the left ventricular wall motion has recovered after the surgeon re-bypassed the bypass [10]. (2) The weight of newborns with congenital heart disease is too low to insert a transesophageal probe for examination. Inserting a transesophageal probe can significantly increase the airway pressure of newborns and some infants and cause pronounced vagal reflexes such as heart rate slowing. (3) $\mathrm{Pa}-$ tients who have difficulty in inserting the probe through the esophagus due to conditions such as laryngeal mask ventilation and separate tracheal intubation in both lungs. (4) Patients with hepatitis or syphilis and other blood-borne diseases: directly inserting the probe through the esophagus may easily lead to cross-infection. In addition, epicardial ultrasound can reduce pharyngeal bleeding and other complications of transesophageal ultrasound. Reduce the adverse effects of inserting the transesophageal probe on the tracheal intubation and esophagus.

Traditional transmyocardial ultrasound generally uses an ordinary transthoracic-phased array transthoracic probe which will be directed by a surgeon after thoracotomy. This method requires the surgeon's ability to operate the probe to display the cut surface very high. Due to the influence of the thoracic surgical incision, the inspection section and angle are greatly restricted, and it is not easy to obtain a clear and stable image due to the most obvious pulsation of the front wall of the heart. At the same time, it is easy to increase the probability of arrhythmia during the probe pressure examination. Because the transesophageal probe is softer, it reduces the pressure on the myocardium, which helps to get closer to the surface of the heart when the heart is beating 


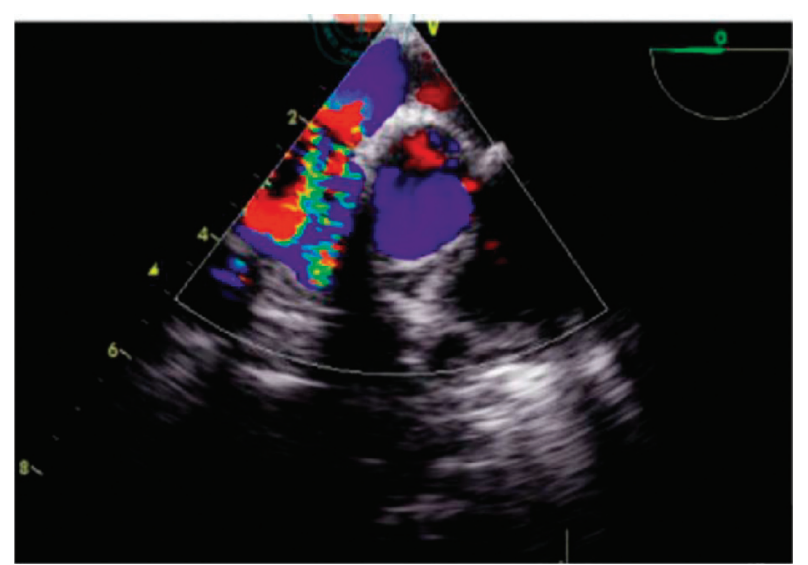

Figure 2: Color Doppler shows that, after the tricuspid valve is deformed, the short-axis view of the aorta shows that the tricuspid valve still has a moderate amount of regurgitation.

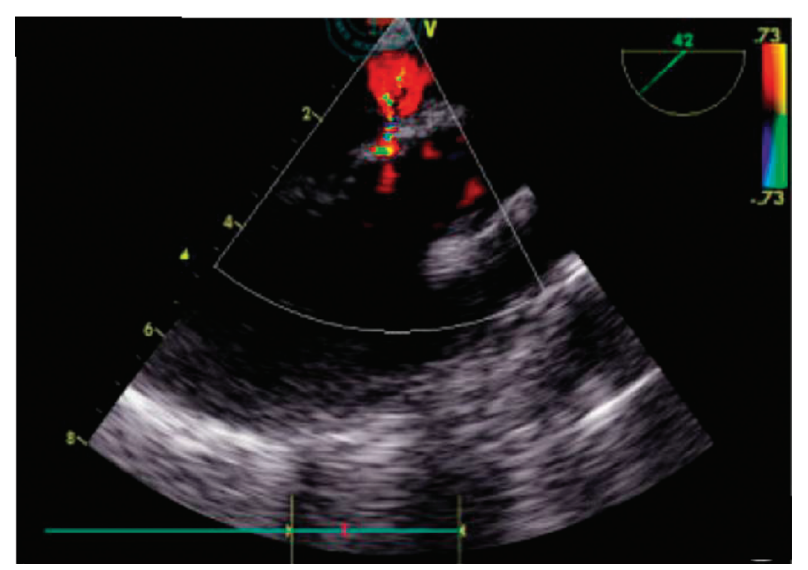

Figure 3: Color Doppler left ventricular short-axis view showing a small amount of residual shunt after ventricular septal defect repair.

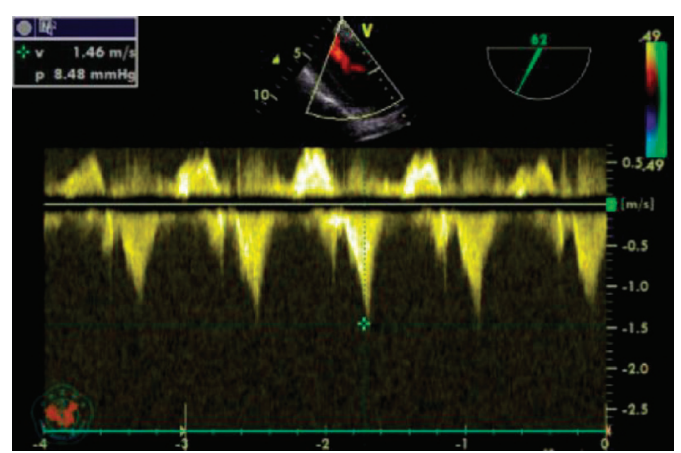

FIgURE 4: Two-dimensional ultrasound left ventricular long-axis level showing blatant obstruction of the left ventricular outflow tract in patients with hypertrophic obstructive cardiomyopathy.

and improves the image quality. At the same time, since the transesophageal probe is relatively small, it is attached to the heart through subtle "flexion," "extension," and "lateral rotation" adjustments of the probe. Even if the anterior chest incision is small, the probe can be placed on the heart's front, side, or diaphragmatic surface. This allows the sonographer who operates the instrument to adjust the rotation angle of the probe wafer, which significantly improves the diversity of the display section. This can meet the requirements of surgery for ultrasound images. The operability of the method is also improved because the requirements to operate the probe are simplified. Physicians can choose pediatric and adult transesophageal probes according to the size of the patient's heart and the purpose of observation. Pediatric transesophageal probes have a higher frequency and more explicit images.

Moreover, the probe is smaller, which is more suitable for children and adults with smaller hearts. However, the penetrating power of adult patients with enlarged hearts is limited during surgery. At this time, adult transesophageal probes need to be selected.

Some scholars have confirmed the value of epicardial ultrasound in the immediate evaluation of valvular surgery. The esophageal probe is placed on the surface of the heart, and the shape and activity of each valve can be well displayed through flexible multiangle rotation. Combining multiple views can also clearly display the poorly displayed pulmonary valve and tricuspid valve through the esophageal probe, which can more accurately determine the valve regurgitation, evaluate the change of valve flow rate, and estimate the pressure difference. It can also show the functional status of the chordae and papillary muscles at the same time. In this study, a patient with tricuspid valve depression deformity showed a clear view of the midvalve regurgitation through the right ventricular inflow tract immediately after the orthosis. The regurgitation was significantly reduced after another turnaround operation. The treatment effect is satisfactory. In another patient with mitral valvuloplasty, the left ventricular long axis and the apical four-chamber view clearly showed that the valve had low or medium regurgitation. The regurgitation disappeared after the second turn of the operation.

Transesophageal probe for epicardial ultrasound has more evident advantages in children with congenital diseases. In addition to 2 cases of residual shunts found in this group of studies, 2 cases of pulmonary atresia with intact ventricular septum were inserted into the balloon through the right ventricular outflow tract under the guidance of transesophageal probe epicardial ultrasound after thoracotomy. We perform a real-time evaluation of the expansion effect after expanding the pulmonary valve with membranous atresia. Observe the condition of the arterial catheter and pulmonary valve regurgitation. Real-time evaluation of left ventricular function and mitral regurgitation at different degrees of ring constriction provides a basis for determining the degree of ring constriction. Especially, in determining residual shunt and valve regurgitation, it is more rapid, sensitive, and accurate.

At present, there are few cases and disease types of transesophageal probes undergoing epicardial ultrasonography. Technically, in-depth research and standardization are also needed. There are also many problems for further study. For example, how can the probe be placed to display the cut surface better? Which cuts should be shown in 
children with different congenital heart diseases and different operations to be more targeted? There have been studies on applying three-dimensional transthoracic probes to epicardial ultrasound examinations. This proves that it provides essential information on morphology and spatial structure in valvular disease and congenital heart disease.

\section{Conclusion}

The intraoperative ultrasound monitoring in cardiac surgery is still mainly based on transesophageal ultrasound, but transepicardial ultrasound overcomes many limitations due to it. It still has an irreplaceable role in certain heart operations, and it can better meet clinical requirements with the advancement of technology. In this study, the use of transesophageal probe for epicardial ultrasonography has the advantages of being more accurate, simple, flexible, and economical.

\section{Data Availability}

The data used to support the findings of this study are available from the corresponding author upon request.

\section{Conflicts of Interest}

The authors declare that there are no conflicts of interest.

\section{References}

[1] A. Beckmann, R. Meyer, J. Lewandowski, A. Markewitz, and J. Gummert, "German heart surgery report 2019: the annual updated registry of the German society for thoracic and cardiovascular surgery," The Thoracic and Cardiovascular Surgeon, vol. 68, no. 4, pp. 263-276, 2020.

[2] A. Beckmann, R. Meyer, J. Lewandowski, A. Markewitz, and W. Harringer, "German heart surgery report 2018: the annual updated registry of the German society for thoracic and cardiovascular surgery," The Thoracic and Cardiovascular Surgeon, vol. 67, no. 5, pp. 331-344, 2019.

[3] H. Brun, R. A. B. Bugge, L. K. R. Suther et al., "Mixed reality holograms for heart surgery planning: first user experience in congenital heart disease," European Heart Journal - Cardiovascular Imaging, vol. 20, no. 8, pp. 883-888, 2019.

[4] K. W. D. Stern, S. M. Emani, G. J. Peek, T. Geva, and S. Kutty, "Epicardial echocardiography in pediatric and congenital heart surgery," World Journal for Pediatric and Congenital Heart Surgery, vol. 10, no. 3, pp. 343-350, 2019.

[5] W. Boettcher, F. Dehmel, M. Redlin, N. Sinzobahamvya, and J. Photiadis, "Cardiopulmonary bypass strategy to facilitate transfusion-free congenital heart surgery in neonates and infants," The Thoracic and Cardiovascular Surgeon, vol. 68, no. 1, pp. 002-014, 2020.

[6] S. R. Fuchs, A. H. Smith, S. L. Van Driest, K. F. Crum, T. L. Edwards, and P. J. Kannankeril, "Incidence and effect of early postoperative ventricular arrhythmias after congenital heart surgery," Heart Rhythm, vol. 16, no. 5, pp. 710-716, 2019.

[7] M. Çelik, F. Aygün, and M. Özkan, "Sternotomy with electrocautery and sternal wound infection in congenital heart surgery in patients under 1 year of age," Journal of Cardiac Surgery, vol. 36, no. 7, pp. 2336-2341, 2021.
[8] T. Abe, K. Nakano, N. Hirahara, N. Motomura, H. Miyata, and S. Takamoto, "Current status of cardiovascular surgery in Japan: analysis of data from Japan cardiovascular surgery database in 2015, 2016. 3-v," General thoracic and cardiovascular surgery, vol. 67, no. 9, pp. 742-749, 2019.

[9] C. Bolcal, M. Kadan, H. Sicim, M. Ulubay, and V. Yildirim, "Redo robotic cardiac surgery and concomitant cesarean section in a pregnant patient with dextrocardia and situs inversus totalis," Journal of Cardiac Surgery, vol. 34, no. 9, pp. 863-866, 2019.

[10] A. Harky, G. Chaplin, J. S. K. Chan et al., "The future of open heart surgery in the era of robotic and minimal surgical interventions," Heart Lung \& Circulation, vol. 29, no. 1, pp. 49-61, 2020. 